\title{
Evaluation of functional disability and pain in patients with chronic low back pain submitted to physiotherapy
}

\author{
Andrei Luiz Sales Teixeira( ${ }^{(1)}$, Noberto Fernandes da Silva(2), Edson Meneses da Silva Filho(3)
}

\begin{abstract}
Introduction: Chronic low back pain (CLBP) is one of the causes that usually result in mobility limitations in the economically active population due to musculo-skeletal disorders. It has, as main symptoms, pain and disability and should be seen as a public health problem. Objective: This study aimed to evaluate the Functional Disability (FD) and pain intensity in patients with CLBP, underwent to manipulative therapy and conventional physiotherapy at Getulio Vargas Hospital. Method: This was a randomized trial study with a control group, with a sample, participants with CLBP, who were divided into two groups: those who underwent manipulative physiotherapy (group 1) and those who underwent conventional therapy (group 2), and the both groups were subjected to two treatments at different times. The program lasted five weeks, and 2 weeks for each intervention, performing two weekly sessions, which average duration was 30 minutes and one week interval between treatments. There were collected sociodemographic data, data related to FD using the Disability questionnaire Roland-Morris (RMDQ) and data related to pain intensity using the Visual Analogue Scale (VAS). Results: The study included 14 patients with mean age of $51+9$ years. They showed significant improvement in $R M D Q(p<0.001)$ and VAS ( $p<0.001)$, after being subjected to manipulative group, also showing a significant treatment effect over conventional treatment in both RMDQ $(p=0.004)$ and EVA $(p=0.006)$. Conclusion: Through this study, it can be shown that patients with CLBP showed significant improvements in functional capacity, by reducing the FD and reduction of painful symptoms, after being subjected to a five-week-program of manipulative physiotherapy.
\end{abstract}

KEY WORDS: Manual therapy; Disability; Manipulation

\section{INTRODUCTION}

The pain in the spine is one of the causes most frequently found in the economically active population due to muscleskeletal disorders and is associated with physical problems, inadequate postures and stressful repetitive movements ${ }^{(1)}$, and $80 \%$ of these changes affect the region low back. ${ }^{(2)}$ It is believed that about $50 \%$ to $80 \%$ of the population are or will be affected by back pain or low back pain at some point in life ${ }^{(3,4)}$ and of these $90 \%$ will present recurrence. ${ }^{(5)}$

This pain also occurs due to factors such as homeostasis breakdown, secondary to mechanical blockages that result in restriction of range of motion, muscle spasms, with consequent protective postural alterations and decrease of muscular trophism, leading to functional limitations or incapacities for the activities development of daily life as well as causing restrictions on the individual's participation in society. ${ }^{(6,7)}$

Although pain complaint is the main symptoms presented, chronic back pain (CBP) is seen in the literature as multifactorial ${ }^{(4)}$ and it is estimated that in $95 \%$ of cases, the cause of complaints is associated with a degenerative process in the intervertebral disc, as the herniated lumbar disc $\mathrm{c}^{(7)}$. It is believed that in only $10 \%$ of cases, the cause of CBP is identified by health professionals. ${ }^{(8.9)}$

Despite the low identification of CBP there are several forms of treatment that aim to help these patients, for example, conservative approaches which include medication and conventional physiotherapy (CP). ${ }^{(9)}$ Physical therapy uses as physical therapy resource, therapeutic exercises in an attempt to reduce pain intensity, increase mobility and function, gain resistance and muscle strength, reduce the time of dysfunction and medical care. ${ }^{(3)}$ However, the understanding of the lesion process and the use of results of the evaluation process are essential to proper development of physical therapy care plan and optimization of the results. ${ }^{(10)}$

Among the approaches that aim to understand the lesion process is the manipulative physiotherapy (MP), and within this modality is the osteopathic manipulation, which has been used as treatment of choice for CPB by incorporating manipulative approaches, with high speed execution and low impact, aimed at finding and solving problems of body 
unity, with few sessions, low cost, improving mechanisms of homeostasis, as well as the interrelationships between the structure of the body, guaranteeing good tissue nutrition, restoring nerve and vascular flow. ${ }^{(11-13)}$

The MP is a form of treatment that seems to be effective for CBP because it acts directly on the cause of the problems through manipulations, allowing to achieve a normalization of the patient's physiological structures faster, causing people to improve the functionality of the movements and decrease of painful symptoms, requiring less conservative interventions.

Considering the efficacy of MP and $\mathrm{CP}$ in the treatment of $C B P$, the present study had as objective to evaluate the functional incapacity and pain intensity in patients with CBP, submitted to manipulative physiotherapy and conventional physiotherapy in the Getúlio Vargas Hospital.

\section{METHOD}

The present study was a randomized clinical trial with a control group, performed at the clinic of the Center for Rehabilitation and Physical Rehabilitation of the Hospital Getúlio Vargas (HGV), Recife/PE, from October to December 2015.

It was considered as inclusion criteria: patients of both genre; Age from 30 to 59 years, with diagnosis of low back pain present for more than 3 months; sedentary than which do not present prior physical therapy history to at least 3 months. Exclusion criteria were: cognitive deficiency, auditory or visual impairment which compromises the response to the questionnaires used in the present study; Presence of root symptoms or changes in the neuro-muscle-skeletal apparatus which prevent the performance of exercises and/or manipulation, history of previous surgeries in the spine; Presence of degenerative bone-muscular diseases or any other disease affecting the spine, other than those that develop with low back pain; Presence of tumors, infectious diseases, prosthetics, respiratory diseases, umbilical hernia, lower limb discrepancy, pregnant or any other contraindication to manipulative physical therapy.

Participated in this study, patients with clinical and functional diagnosis of low back pain of both genres, who were referred by the trauma-orthopedic outpatient clinic of the Getúlio Vargas Hospital of Recife-PE, and were willing to contribute with the research during the period of data collection. After clarification, the volunteers provided their consent to participate in the study, by signing a Free and Informed Consent Term, in accordance with Resolution 466/09 of the National Health Council, of the Ministry of Health (CNS/MS). The study was approved by the Research Ethics Committee of the University of Pernambuco (Protocol No. 078765/15).

The individuals selected were randomized, and placed in 2 dark envelopes, sealed and labeled as "group 1" and "group 2" by a subject blinded to the study, ensuring a hidden allocation of participants. Randomization was done through the site https://www.random.org/sequences/. Participants were represented by numbers from 1 to 28 in the part of generation of random sequences from the site. Randomization was generated in 2 columns, which represented each group.

All were assigned to one of two groups: those who underwent manipulative physiotherapy (group 1) and those who underwent conventional therapy (group 2), and the two groups were subjected to two treatments at different times. All participants underwent intervention for both physiotherapy techniques, but at different times, leaving a 1-week interval between the 2 interventions (wash-out period), which were applied to the participants, prior to the beginning of each program and to the end, the RMDQ questionnaire and the EVA scale. The program had duration of 5 weeks of treatment, 2 weeks for each intervention and 1 week of wash-out, with two weekly sessions, with an average duration of 30 minutes each. After the interventions, the results were analyzed by a researcher not involved in the study.

In the group 1, patients were rehabilitated by manipulation of the thoracic region, an osteopathic technique known as "Dog Technic", in which the patient is placed in the supine position on the stretcher with arms folded over his or her own body, hugging itself, afterwards the researcher places the dominant hand in the thoracic region involving the patient against its thoracic, ending the technique with the manipulation through the "body drop". The sacroiliac region was manipulated by the "lumbar roll" technique, which the patient lies in lateral decubitus position with the leg that is in contact with the stretcher, stretched and the leg that is above, in hip and knee flexion, after that the researcher rotates the upper extremity of the patient towards the lower limb which is above, then the investigator places the forearm in the region which he intends to manipulate the sacrum by leveraging the lower limb which is previously flexed.

In group 2, the patients underwent a therapeutic program of self-passive stretching for lumbar spine with a total duration of 5 minutes and active exercises of flexion-extension, Abduction-Adduction and extension for lower limbs in open kinetic chain ( 3 sets of 12 repetitions each), performed in the supine position, in lateral decubitus and pronated respectively, in a stretcher. After the re-evaluation, the patients in group 1 became group 2 and vice versa, and the same procedures described previously were performed.

All were assessed for socio-demographic and health data such as: name, age, gender, date of assessment, marital status, education and current occupation level. For the evaluation of the IF, the Roland-Morris Functional Disability Questionnaire (RMDQ) was used. ${ }^{(14)}$ And for evaluation of pain intensity was used the Visual Analogue Scale. ${ }^{(15)}$ The whole collection was made by a trained and blind researcher on the allocation of participants. 
The RMDQ is a specific questionnaire to measure the degree of functional incapacity of patients with low back pain and validated in Brazil. It is composed by 24 questions related to activities of daily living, pain and function. The questions have a dichotomous answer (yes or no) and for each affirmative question is assigned 1 point. The score is the sum of the values, being able to obtain a minimum score of " 0 " and a maximum score of "24". This questionnaire has a cutoff score of " 14 ", that is, individuals evaluated with a score equal to or greater than "14" are classified as functionally incapacitated. ${ }^{(14)}$

The Visual Analogue Scale (VAS) is a scale used to quantify the intensity and perception of the individual about their pain, being graded according to ordinal scores: no pain (0), mild ( 1 to 2 ), moderate ( 3 to 5 ) and intense ( 6 to 10 ). The patient was clarified on the scale using visual scale numbered from zero to ten, where the pain intensity was placed according to the patient's sensation. ${ }^{(15)}$

Initially, the patients were characterized by the frequency distribution as to gender and sociodemographic characteristics, and in the case of age, the mean was presented with their respective standard deviation, as well as when groups were compared according to the scale of functional disability and scale the intensity of pain. In the intra-group comparison, the
T test was applied for paired samples and for the comparison of the effect of the groups the MANOVA test was applied for repeated measures, using as factor the groups of patients submitted to manipulative and conventional physiotherapy.

The distribution of the RMDQ and FAS scores were assumed to have normal distribution and the parametric methodology was used for the hypothesis tests. The tests were considered with a significance level of $p<0.05$. The software used in the analysis was Stata version 12.0.

\section{RESULTS}

The sample initially evaluated was of 56 patients, of whom 14 were eligible, and all of them were submitted to manipulative and conventional physiotherapy (Figure 1).

Half of patients surveyed were male, with a mean age of $51+9$ years, minimum age of 34 and maximum of 59 years. From 14 involved in the study, $78.6 \%$ were married and according to schooling, $57.2 \%$ had incomplete elementary education and $35.7 \%$ had completed high school. Half of the patients had income between 1 and 2 minimum wages, only one patient reported being unemployed, and the others had employment, 7 of whom were self-employed and 6 had a fixed job (Table 1).

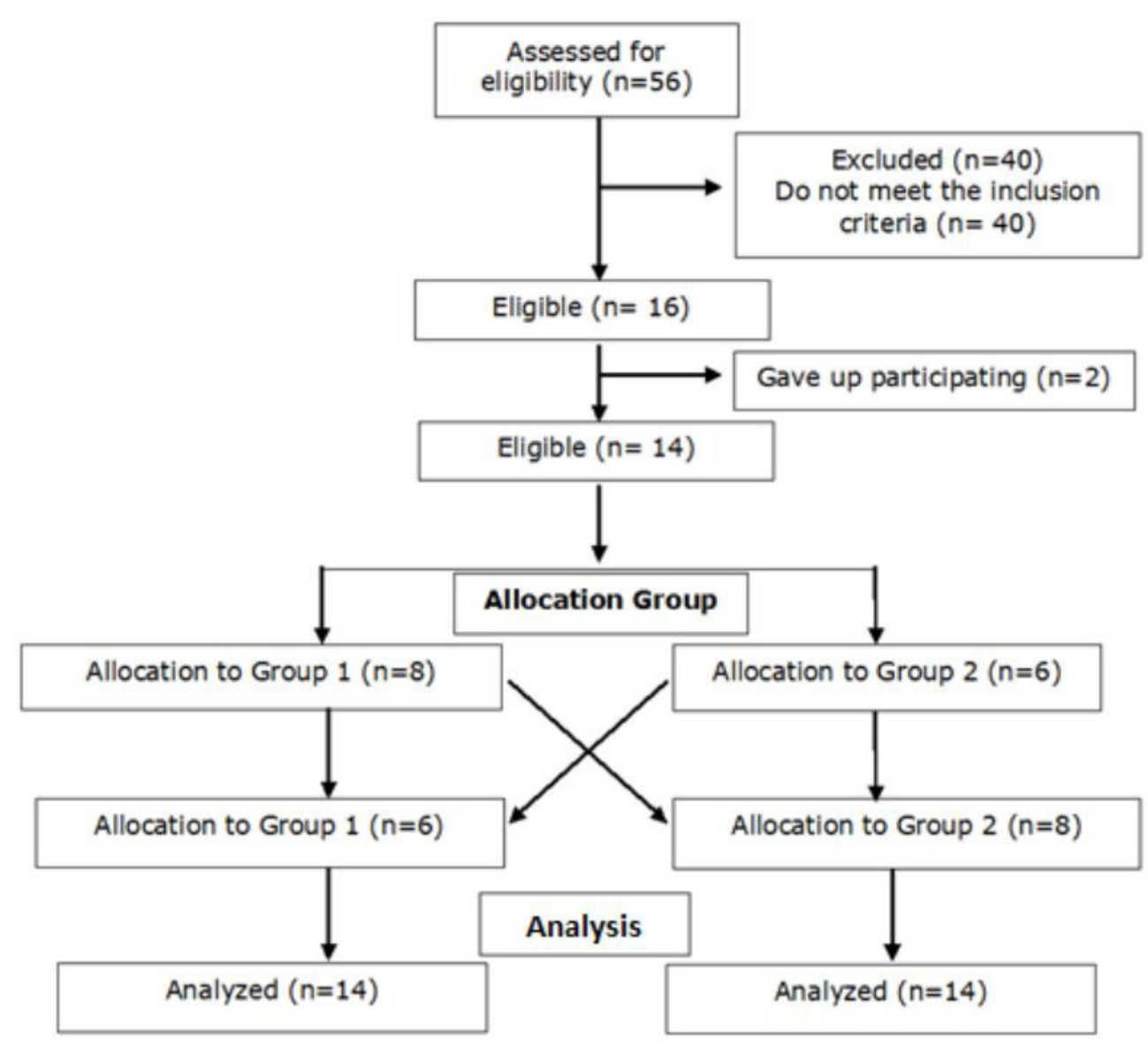

Figure 1. Flowchart of the study. 
The functional disability score RMDQ shows that the group of patients submitted to manipulative treatment had an average reduction of 10.0 points, whereas in the group that received conventional physiotherapeutic treatment there was

Table 1. Characteristics of patients with chronic low back pain undergoing manipulative and conventional physical therapy, treated at outpatient Rehabilitation Center and Rehabilitation Hospital Physical Getulio Vargas, Recife/PE.

\begin{tabular}{lc}
\hline \multicolumn{1}{c}{ Characteristics } & Statistics \\
\hline Number of respondents & 14 patients $(100 \%)$ \\
Age (mean \pm standard deviation) & $51 \pm 9.0$ years \\
Genre & \\
$\quad$ Male & $7(50.0 \%)$ \\
$\quad$ Female & $7(50.0 \%)$ \\
Marital status & \\
$\quad$ Married & $11(78.6 \%)$ \\
$\quad$ Not married & $2(14.3 \%)$ \\
Widower & $1(7.1 \%)$ \\
Schooling & \\
Incomplete 1st grade & $8(57.2 \%)$ \\
Complete 1st grade & $1(7.1 \%)$ \\
Incomplete 2nd grade & $5(35.7 \%)$ \\
Income (in minimum salaries) & \\
Less than 1 & $5(35.7 \%)$ \\
From 1 to 2 & $7(50.0 \%)$ \\
More than 2 & $2(14.3 \%)$ \\
Occupation & \\
Unemployed & $1(7.1 \%)$ \\
Self Employed & $7(50.0 \%)$ \\
Employed & $6(42.9 \%)$ \\
\hline
\end{tabular}

an average reduction of 2.5 points. There was also a significant reduction in mean functional disability scores after therapy in the manipulative group (intra-group analysis) $(p<0.001)$. When the treatment effect between the groups was tested, there was a significant statistical significance $(p=0.013)$, which suggests a greater gain in functional capacity in the group of patients in which underwent manipulative physical therapy (table 2).

A similar result is observed when the pain intensity scale is analyzed. In the group of patients submitted to manipulative treatment there was an average reduction of 3.64 points, while in the group that received traditional physiotherapy treatment the mean reduction was 0.85 points, however, there was a significant reduction in the mean of the scale score of pain intensity after therapy, only in the manipulative group (intra-group analysis) ( $p<0.001)$. Thus, testing the effect between the groups, there was statistical significance $(p=0.006)$, which also suggests a greater reduction of pain intensity in the group of patients which underwent manipulative physical therapy (Table 3).

\section{DISCUSSION}

This study observed a significant reduction in the functional disability scores of the RMDQ $(p<0.001)$ and painful FAS symptoms $(p<0.001)$ for the manipulative group, as well as a better treatment effect of this group over the conventional group, as in the RMDQ $(p=0.004)$ as in EVA $(p=0.006)$, showing that the Manipulative Physiotherapy (MF) presented better results when compared with conventional physiotherapy (CP). Similar studies that performed a 4-week study comparing MF to CP showed positive results regarding functional capacity and pain. ${ }^{(16,17)}$

These improvements are in accordance with some Osteopathy principles which consider that homeostasis can

Table 2. Average of results obtained through functional disability scale RMDQ before and after manipulative physiotherapy (a) and before and after conventional therapy (b) of patients with chronic low back pain treated at the clinic of the Rehabilitation Center and Rehabilitation Physics Getulio Vargas hospital, Recife/PE.

\begin{tabular}{lccc}
\multicolumn{1}{c}{ Groups } & Before (mean \pm SD) & After (mean \pm SD) & p-value \\
\hline Manipulative Physiotherapy ${ }^{\mathrm{a}}(\mathrm{n}=14)$ & $17.6 \pm 4.8$ & $7.6 \pm 3.9$ & $<0.001^{*}$ \\
Conventional physiotherapy $^{\mathrm{a}}(\mathrm{n}=14)$ & $16.3 \pm 6.2$ & $13.8 \pm 4.1$ & 0.120 \\
Efeito dos grupos $^{\mathrm{b}}$ & & & $0.004^{*}$ \\
\hline
\end{tabular}

RMDQ - Roland Morris Disability Questionary

a $t$ Test for paired samples

${ }^{b}$ MANOVA with a factor for repeated measures

* Statistically significant difference $(p<0.05)$

Table 3. Average score obtained by visual analogue scale of pain intensity before and after manipulative physiotherapy (a) and before and after conventional therapy (b) of patients with chronic low back pain treated at the outpatient Rehabilitation Center Rehabilitation and Physical the Getulio Vargas Hospital, Recife/PE.

\begin{tabular}{lccc}
\hline \multicolumn{1}{c}{ Groups } & Before (mean \pm SD) & After (mean \pm SD) & p-value \\
\hline Manipulative Physiotherapy ${ }^{\mathrm{a}}(\mathrm{n}=14)$ & $7.35 \pm 2.06$ & $3.71 \pm 2.05$ & $<0.001^{*}$ \\
Conventional physiotherapy $^{\mathrm{a}}(\mathrm{n}=14)$ & $6.92 \pm 2.23$ & $6.07 \pm 1.21$ & 0.212 \\
${\text { Effect } \text { of groups }^{\mathrm{b}}}$ & & $0.006^{*}$ & \\
\hline
\end{tabular}

a test for paired samples

${ }^{b}$ MANOVA with a factor for repeated measures

Statistically significant difference $(p<0.05)$ 
be altered through biochemical, bioelectrical, neuroendocrine and/or sympathomimetic mechanisms affecting specific structures or receptors. In CBP patients, pain mechanisms encompass a complex matrix of different processes such as biomechanical, genetic, neurophysiological and/or psychological factors, each capable of contributing to clinical manifestations and symptoms. ${ }^{(13)}$

However, it is believed that the mechanism of action of $\mathrm{MP}$ is related to an attenuation of the sensory input of the afferent muscle spindle, which reduces reflex contractile activity, leading to normalization of muscle activity through reduction of activity on the hyperactive side and increase of activity in the hypoactive side. This may be justified by the potential of a manipulation to interrupt the pain-spasm cycle by negative regulation of central sensitization associated with clinical status. ${ }^{(18,19)}$

Another hypothesis is that the improvement of the symptoms after manipulative treatment in these patients could be related to a greater kinetic balance of the muscles and articulations of the spine, restoring joint bad positioning and reducing inflammation around the neural root. ${ }^{(20)}$

On the inflammatory process generated by the $C B P$, there are studies that analyze other aspects, showing correlations of cytokine concentrations with clinical measures of manipulative treatment, where were found associations between IL-1 $\beta$ and IL- 6 concentrations and the number of key osteopathic lesions and between IL- 6 and LBP and severity at baseline of the study. However, only TNF- $\alpha$ concentration showed a significant reduction after 12 weeks, in response to manipulation when compared to patients receiving mock manipulation. ${ }^{(21)}$

Despite the benefits presented by the authors about MP, for its effectiveness, treatment plans should be formulated, with approaches based on osteopathic principles, in different patients with similar symptoms and may require plans to concentrate in several locations of the spine, including above and infraspinatis portions, from the possibility that a manipulation can affect the segments function in distal levels of the manipulated segment ${ }^{(19)}$, as proposed in our study.

This manipulative treatment might be focused on improving function, by reducing peripheral nociception and central facilitation, in order to enable individuals to move and resume their normal activities by improving functional capacity. When osteopathic principles are actively applied in practice to create a treatment plan for a CBP patient, the result is a personalized and effective plan of care usually combining non-pharmacological treatment strategies. ${ }^{(13)}$

Thus, it is verified in our investigation that, with few treatment sessions using MP, satisfactory responses of the reduction of functional disability and pain intensity can be achieved in the short term, it is worth noting that some authors corroborate these findings. ${ }^{(22.23)}$
However, there are concerns in the literature that $\mathrm{CBP}$ is often managed with expensive treatments and of questionable long-lasting effectiveness. (24) Our results support the effectiveness and safety of MP, however, it does not address its long-term cost-effectiveness.

A meta-analysis concluded that manipulative physical therapy significantly reduced chronic low back pain for at least three months and could extend it up to one year. ${ }^{(25)}$ Thus, a larger study is warranted to evaluate the efficacy and cost-effectiveness of therapy to be addressed in the long term in the future, increasing the relevance for the outcome of treatment.

Attempts have been made to ensure that the present study has a low risk of bias, including appropriate procedures for randomization, blinding, concealed allocation, and similarity at the start of the study. In contrast, it was not possible to blind the therapist, due to the nature of the interventions, which does not eliminate the risk of bias.

Therapist blindness is not feasible in trials with active treatment interventions, such as exercise or manipulation, or ethical as requirements for a given information record. Therefore, the lack of masking of the therapist could be interpreted as a limitation of the study.

Other limitations of this study include the recruitment of patients seeking physical therapy through the medical staff of the hospital, the lack of a placebo or no treatment group, and a selection of patients with acute pain, in addition to the sample size associated with poor adherence, possibly due to the low socioeconomic level and distance from the center where the program was developed and due to the structural problems faced by the hospital, reducing the number of study participants. These factors may have limited the response of these interventions.

In addition, it should take care with the results of this study as they are restricted to a short-term program. In addition, the present study used a combination of only 2 physiotherapy techniques, making it difficult to know whether these techniques alone are sufficient to achieve prolonged effects, or whether there is a necessity for a combination of techniques to achieve the observed effects; Therefore, it is not known if these effects and mechanisms of action of the techniques are maintained over a longer period of time. In addition, there is a necessity for a more expressive number of participants, so new studies must be produced with long-term treatment in order to these results to be more significant and elucidate the impacts of treatment effects.

\section{CONCLUSION}

Through this study, it can be demonstrated that patients with CBP present functional capacity improvement, by reducing the IF and reducing pain symptoms through the use of manipulative physiotherapy (MP) and physical therapy (PT), and the results presented by MP treatment were more significant. 
We highlight the relevance of the design of new studies on CBP that may include a larger quantity of osteopathic and conventional techniques with a prolonged period of treatment and reassessment, in order to obtain a more accurate scenery of the efficiency of the best physiotherapeutic treatment for the pathology.

\section{CONFLICT OF INTEREST}

The authors report that there is no conflict of interest

\section{AUTHOR DETAILS}

${ }^{1}$ Physiotherapist, Residency Program Resident in Urgency, Emergency and Trauma of Hospital Getúlio Vargas, Timbaúba (PE), Brazil.

${ }^{2}$ Physiotherapist, Hospital Getúlio Vargas, Timbaúba (PE), Brazil.

\section{REFERENCES}

1. Nogueira HC, Navega MT. Influência da escola de postura na qualidade de vida, capacidade funcional e intensidade de dor e flexibilidade de trabalhadores administrativos. Fisio. e Pesq., São Paulo, 2011;18(4):353-8.

2. Mancin B, Bonvicine $G$, Gonçalves $C$, Barboza MAI. Análise da influência do sedentarismo sobre a qualidade de vida de pacientes portadores de dor lombar crônica. Conscientia e Saúde. 2008;7(4):441-7.

3. Adorno MLGR, Brasil-neto JP. Avaliação da qualidade de vida com o instrumento SF-36 em lombalgia crônica. Acta Ortop Bras. 2013;21(4):2027.

4. Yang $\mathrm{M}$ et al. Effectiveness of Chinese massage therapy (Tui $\mathrm{Na}$ ) for chronic low back pain: study protocol for a randomized controlled trial. Trials. 2014 Oct 29;15:418.

5. Ferreira S, Pereira MG. Preditores da qualidade de vida e incapacidade functional em doentes com lombalgia crônica em tratamento diferenciado. Rev. SBPH 2011;14(1).

6. Mascarenhas CHM, Santos LS. Avaliação da dor e da capacidade funcional em indivíduos com lombalgia crônica. J Health Sci Inst. 2011;29(3):205-8.

7. Zdrodowska B, Leszczyńska-Filus M, Leszczyński R, Błaszczyk J. Comparison of the effect of laser and magnetic therapy for pain level and the range of motion of the spine of people with osteoarthritis lower back. Pol Merkur Lekarski. 2015 Jan;38(223):26-31.

8. Balagué F, Mannion AF, Pellisé F, Cedraschi C. Non-specific low back pain. Lancet 2012;379:482-491.

9. Xia T et al. Study protocol for patient response to spinal manipulation - a prospective observationalclinical trial on physiological and patientcentered outcomes in patients with chronic low back pain. BMC Complement Altern Med. 2014 Aug 8;14:292.

10. Tozzi P, Bongiorno D, Vitturini C. Low back pain and kidney mobility: local osteopathic fascial manipulation decreases pain perception and improves renal mobility. Journal Bodyw Mov Ther. 2012 Jul;16(3):381-91.
11. Oliveira RF et al. Immediate Effects of Region Specific and Non Region Specific Spinal Manipulative Therapy in Randomized Patients With Chronic Low Back Pain: A Controlled Trial. PHYS THER. 2013;93:748-756.

12. Voogt $L$ et al. Analgesic effects of manual therapy in patients with musculoskeletal pain: A systematic review. Manual Ther 2015;20:250-256.

13. Kuchera ML. Applying Osteopathic Principles for Treatment of Patients With Chronic Pain. Journal Am Osteopath Assoc. 2007;107(suppl 6):ES28ES38.

14. Monteiro J, Faísca L, Nunes $\mathrm{O}$, Hipólito J. Questionário de incapacidade de roland morris-adaptação e validação para os doentes de língua portuguesa com lombalgia. Acta Med Port 2010;23:761-766.

15. Toniolli ACS, Pagliuca LMF. Tecnologia tátil para a avaliação da dor em cegos. Rev Latinoam Enferm. 2003;11(2):220-6.

16. Cruser A et al. A randomized, controlled trial of osteopathic manipulative treatment for acute low back pain in active duty military personnel. J of Manual and Manipulative Ther 2012 VOL. 20 No. 1.

17. Hougt E, Stephenson R, Swift L. A comparison of manual therapy and active rehabilitation in the treatment of non specific low back pain with particular reference to a patient's Linton \& Hallden psychological screening score: a pilot study. BMC Musculoskeletal Disorders 2007, 8:106.

18. Clark BC et al. Muscle functional magnetic resonance imaging and acute low back pain: a pilot study to characterize lumbar muscle activity asymmetries and examine the effects of osteopathic manipulative treatment. Osteop Med and Primary Care 2009, 3:7.

19. Lehman GJ, Vernon H, and McGill SM. Effects of a Mechanical Pain Stimulus on Erector Spinae Activity Before and After a Spinal Manipulation in Patients With Back Pain: A Preliminary Investigation. Journal of Manipulation and Physio Ther July/August 2001 V 24 N 6.

20. Zhang W et al. Therapeutic Effects of Chinese Osteopathy in Patients with Lumbar Disc Herniation. The American J. of Chin. Med., 2013,Vol. 41, No. 5, 983-994.

21. Licciardone JC, Kearns CM, Hodge LM, Bergamini MV. Associations of cytokine concentrations with key osteopathic lesions and clinical outcomes in patients with nonspecific chronic low back pain: results from the OSTEOPATHIC Trial. J Am Osteopath Assoc 2012;112:596 e 605.

22. Licciardone JC, Aryal S. Clinical response and relapse in patients with chronic low back pain following osteopathic manual treatment: Results from the OSTEOPATHIC Trial . Manual Therapy xxx (2014) 1-8.

23. Licciardone $\mathrm{JC}$ et al. Osteopathic Manual Treatment and Ultrasound Therapy for Chronic Low Back Pain: A Randomized Controlled Trial. Ann Fam Med 2013;11:122-129.

24. Deyo RA, Mirza SK, Turner JA, Martin BI. Overtreating chronic back pain: time to back off? J Am Board Fam Med. 2009;22(1):62-68.

25. Licciardone JC, Brimhall AK, King LN: Osteopathic manipulative treatment for low back pain: a systematic review and metaanalysis of randomized controlled trials. BMC Musculoskelet Disord 2005, 6:43. 